\title{
Introducing a New Intrinsic Metric
}

\author{
Oona Rainio® and Matti Vuorinen®
}

\begin{abstract}
A new intrinsic metric called the $t$-metric is introduced. Several sharp inequalities between this metric and the most common hyperbolic type metrics are proven for various domains $G \subsetneq \mathbb{R}^{n}$. The behaviour of the new metric is also studied under a few examples of conformal and quasiconformal mappings, and the differences between the balls drawn with all the metrics considered are compared by both computational and analytical means.
\end{abstract}

Mathematics Subject Classification. Primary 51M10; Secondary 30C65.

Keywords. Hyperbolic geometry, Intrinsic geometry, Intrinsic metrics, Quasiconformal mappings, Triangular ratio metric.

\section{Introduction}

In geometric function theory, one of the topics studied deals with the variation of geometric quantities such as distances, ratios of distances, local geometry and measures of sets under different mappings. For such studies, we need an appropriate notion of distance that is compatible with the class of mappings studied. In classical function theory of the complex plane, one of the key concepts is the intrinsic distance, which measures not only how close the points of the domain are to each other but also how they are located inside the domain with respect to its boundary.

The best-known example of a metric used to study intrinsic distances is the hyperbolic metric, which is also the foundation of the classical hyperbolic geometry. It has several desirable analytical properties but is often very difficult to define in subdomains $G$ of an arbitrary metric space $X$. Because of this issue, several other newer, more general versions of the hyperbolic metric have been introduced. These generalizations share some but not all intricate features of 
the hyperbolic metric and, in particular, they take into account the location of the points inside the domain. Furthermore, the closures of the balls defined with most of these metrics never intersect the boundary of the domain.

In our research, we call such a generalization of the hyperbolic metric that fulfills all the properties listed in [7, p. 79] a hyperbolic type metric and any metric whose values are affected by the boundary of the domain an intrinsic metric, regardless whether it has also the other properties of a hyperbolic type metric or not. Note that these terms might have slightly different meanings in the literature. More potential properties for a hyperbolic type metric are presented in [7, p. 191-192]. Examples of a hyperbolic type metric include the triangular ratio metric studied in [12-14], the Barrlund metric [4] and the Cassinian metric [10], whereas this work focuses on the following new intrinsic metric.

Definition 1.1. Let $G$ be some non-empty, open, proper and connected subset of a metric space $X$. Choose some metric $\eta_{G}$ defined in the closure of $G$ and denote $\eta_{G}(x)=\eta_{G}(x, \partial G)=\inf \left\{\eta_{G}(x, z) \mid z \in \partial G\right\}$ for all $x \in G$. The $t$ metric for a metric $\eta_{G}$ in a domain $G$ is a function $t_{G}: G \times G \rightarrow[0,1]$,

$$
t_{G}(x, y)=\frac{\eta_{G}(x, y)}{\eta_{G}(x, y)+\eta_{G}(x)+\eta_{G}(y)},
$$

for all $x, y \in G$.

Here, we mostly concentrate on the special case where $G \subsetneq \mathbb{R}^{n}$ and $\eta_{G}$ is the Euclidean distance.

Unlike the hyperbolic type metrics, the $t$-metric does not have the property about the closed balls not intersecting with the boundary, see Theorem 5.5. This is an interesting aspect since this metric clearly fulfills most of the others, if not all, properties of a hyperbolic type metric in [7, p. 79]. Consequently, we have found an intrinsic metric that is not a hyperbolic type metric by our definition. It is also noteworthy that the $t$-metric has a very simple definition so it could potentially be a great help for researching the distortion under mappings and studying this metric further could solve one old open question, see Conjecture 4.4 and Remark 4.5.

The structure of this article is as follows. In Sect. 3, we prove that the function of Definition 1.1 is really a metric and find the sharp inequalities between this metric and several hyperbolic type metrics, including also the hyperbolic metric, in different domains. In Sect. 4, we show how the $t$-metric behaves under certain quasiconformal mappings and find the Lipschitz constants for Möbius maps between balls and half-spaces. Finally, in Sect. 5, we draw $t$-metric disks and compare their certain properties to those of other metric disks. 


\section{Preliminaries}

In this section, we will introduce the definitions of a few different metrics and metric balls that will be necessary later on but, first, let us recall the definition of a metric.

Definition 2.1. For any non-empty space $G$, a metric is a function $\eta_{G}: G \times G \rightarrow$ $[0, \infty)$ that fulfills the following three conditions for all $x, y, z \in G$ :

(1) Positivity: $\eta_{G}(x, y) \geq 0$, and $\eta_{G}(x, y)=0$ if and only if $x=y$,

(2) Symmetry: $\eta_{G}(x, y)=\eta_{G}(y, x)$,

(3) Triangle inequality: $\eta_{G}(x, y) \leq \eta_{G}(x, z)+\eta_{G}(z, y)$.

Let $\eta_{G}$ be any metric. An open ball defined with it is $B_{\eta}(x, r)=\{y \in$ $\left.G \mid \eta_{G}(x, y)<r\right\}$ and the corresponding closed ball is $\bar{B}_{\eta}(x, r)=\{y \in$ $\left.G \mid \eta_{G}(x, y) \leq r\right\}$. Denote the surface of these balls by $S_{\eta}(x, r)$. For Euclidean metric, these notations are $B^{n}(x, r), \bar{B}^{n}(x, r)$ and $S^{n-1}(x, r)$, respectively, where $n$ is the dimension. In this paper, the unit ball $\mathbb{B}^{n}=B^{n}(0,1)$, the upper half-plane $\mathbb{H}^{n}=\left\{\left(x_{1}, \ldots, x_{n}\right) \in \mathbb{R}^{n} \mid x_{n}>0\right\}$ and the open sector $S_{\theta}=\{x \in \mathbb{C} \backslash\{0\} \mid 0<\arg (x)<\theta\}$ with an angle $\theta \in(0,2 \pi)$ will be commonly used as domains $G$. Note also that the unit basis vectors of $\mathbb{R}^{n}$ will be denoted by $\left\{e_{1}, \ldots, e_{n}\right\}$.

Let us now define the metrics needed for a domain $G \subsetneq \mathbb{R}^{n}$. Denote the Euclidean distance between the points $x, y$ by $|x-y|$ and let $d_{G}(x)=$ $\inf \{|x-z| \mid z \in \partial G\}$. Suppose that the $t$-metric is defined with the Euclidean distance so that

$$
t_{G}(x, y)=\frac{|x-y|}{|x-y|+d_{G}(x)+d_{G}(y)}
$$

for all $x, y \in G$, if not otherwise specified.

The following hyperbolic type metrics will be considered: The triangular ratio metric: $s_{G}: G \times G \rightarrow[0,1]$,

$$
s_{G}(x, y)=\frac{|x-y|}{\inf _{z \in \partial G}(|x-z|+|z-y|)},
$$

the $j_{G}^{*}$-metric: $j_{G}^{*}: G \times G \rightarrow[0,1)$,

$$
j_{G}^{*}(x, y)=\frac{|x-y|}{|x-y|+2 \min \left\{d_{G}(x), d_{G}(y)\right\}},
$$

and the point pair function: $p_{G}: G \times G \rightarrow[0,1)$,

$$
p_{G}(x, y)=\frac{|x-y|}{\sqrt{|x-y|^{2}+4 d_{G}(x) d_{G}(y)}} .
$$

Out of these hyperbolic type metrics, the triangular ratio metric was studied by Hästö [9], and the two other metrics are more recent. As pointed out in [8], the $j_{G}^{*}$-metric is derived from the distance ratio metric found by 
Gehring and Osgood in [6]. Note that there are proper domains $G$ in which the point pair function is not a metric [3, Rmk 3.1 p. 689].

Use notations sh, ch and th for the hyperbolic sine, cosine and tangent, respectively. Define then the hyperbolic metric as

$$
\begin{aligned}
\operatorname{ch} \rho_{\mathbb{H}^{n}}(x, y) & =1+\frac{|x-y|^{2}}{2 d_{\mathbb{H}^{n}}(x) d_{\mathbb{H}^{n}}(y)}, \quad x, y \in \mathbb{H}^{n}, \\
\operatorname{sh}^{2} \frac{\rho_{\mathbb{B}^{n}}(x, y)}{2} & =\frac{|x-y|^{2}}{\left(1-|x|^{2}\right)\left(1-|y|^{2}\right)}, \quad x, y \in \mathbb{B}^{n}
\end{aligned}
$$

in the upper half-plane $\mathbb{H}^{n}$ and in the Poincaré unit ball $\mathbb{B}^{n}[7,(4.8)$, p. $52 \&$ (4.14), p. 55]. In the two-dimensional space,

$$
\operatorname{th} \frac{\rho_{\mathbb{H}^{2}}(x, y)}{2}=\left|\frac{x-y}{x-\bar{y}}\right|, \quad \operatorname{th} \frac{\rho_{\mathbb{B}^{2}}(x, y)}{2}=\left|\frac{x-y}{1-x \bar{y}}\right|=\frac{|x-y|}{A[x, y]},
$$

where $\bar{y}$ is the complex conjugate of $y$ and $A[x, y]=$ $\sqrt{|x-y|^{2}+\left(1-|x|^{2}\right)\left(1-|y|^{2}\right)}$ is the Ahlfors bracket [7, (3.17) p. 39].

Note that the following inequalities hold for the hyperbolic type metrics.

Lemma 2.2. [8, Lemma 2.3, p. 1125] For a proper subdomain $G$ of $\mathbb{R}^{n}$, the inequality $j_{G}^{*}(x, y) \leq p_{G}(x, y) \leq \sqrt{2} j_{G}^{*}(x, y)$ holds for all $x, y \in G$.

Lemma 2.3. [8, Lemma 2.1, p. 1124 \& Lemma 2.2, p. 1125] For a proper subdomain $G$ of $\mathbb{R}^{n}$, the inequality $j_{G}^{*}(x, y) \leq s_{G}(x, y) \leq 2 j_{G}^{*}(x, y)$ holds for all $x, y \in G$.

Lemma 2.4. [7, p. 460] For all $x, y \in G \in\left\{\mathbb{H}^{n}, \mathbb{B}^{n}\right\}$,

$$
\begin{aligned}
\text { (1) } \quad \operatorname{th} \frac{\rho_{\mathbb{H}^{n}}(x, y)}{4} \leq j_{\mathbb{H}^{n}}^{*}(x, y) \leq s_{\mathbb{H}^{n}}(x, y) \\
=p_{\mathbb{H}^{n}}(x, y)=\operatorname{th} \frac{\rho_{\mathbb{H}^{n}}(x, y)}{2} \leq 2 \operatorname{th} \frac{\rho_{\mathbb{H}^{n}}(x, y)}{4} \\
\text { (2) } \quad \operatorname{th} \frac{\rho_{\mathbb{B}^{n}}(x, y)}{4} \leq j_{\mathbb{B}^{n}}^{*}(x, y) \leq s_{\mathbb{B}^{n}}(x, y) \leq p_{\mathbb{B}^{n}}(x, y) \\
\leq \operatorname{th} \frac{\rho_{\mathbb{B}^{n}}(x, y)}{2} \leq 2 \operatorname{th} \frac{\rho_{\mathbb{B}^{n}}(x, y)}{4}
\end{aligned}
$$

\section{3. t-Metric and Its Bounds}

Now, we will prove that the function $t$ is truly a metric in the general case.

Theorem 3.1. For any metric space $X$, a domain $G \subsetneq X$ and a metric $\eta_{G}$ defined in $G$, the function $t_{G}$ is a metric.

Proof. The function $t_{G}$ is a metric if it fulfills all the three conditions of Definition 2.1. Trivially, the first two conditions hold. For all $k>0$ and $x, y \geq 0$,

$$
x \leq y \quad \Leftrightarrow \quad \frac{x}{x+k} \leq \frac{y}{y+k}
$$


and it follows from this and the triangle inequality that

$$
\begin{aligned}
t_{G}(x, y) & =\frac{\eta_{G}(x, y)}{\eta_{G}(x, y)+\eta_{G}(x)+\eta_{G}(y)} \leq \frac{\eta_{G}(x, z)+\eta_{G}(z, y)}{\eta_{G}(x, z)+\eta_{G}(z, y)+\eta_{G}(x)+\eta_{G}(y)} \\
& \leq \frac{\eta_{G}(x, z)}{\eta_{G}(x, z)+\eta_{G}(x)+\eta_{G}(z)}+\frac{\eta_{G}(z, y)}{\eta_{G}(z, y)+\eta_{G}(y)+\eta_{G}(z)} \\
& =t_{G}(x, z)+t_{G}(z, y)
\end{aligned}
$$

for all $x, y, z \in G$. Thus, $t_{G}$ fulfills the triangle inequality, too.

We now show that the method of proof of Theorem 3.1 can be used to prove that several other functions are metrics.

Theorem 3.2. If $G$ is a proper subset of a metric space $X, \eta_{G}$ some metric defined in the closure of $G$ and $c_{G}: G \times G \rightarrow[0, \infty)$ some symmetric function such that, for all $x, y, z \in G$,

$$
c_{G}(x, z) \leq \eta_{G}(z, y)+c_{G}(x, y),
$$

then any function $\phi_{G}: G \times G \rightarrow[0,1]$, defined as

$$
\phi_{G}(x, x)=0, \quad \phi_{G}(x, y)=\frac{\eta_{G}(x, y)}{\eta_{G}(x, y)+c_{G}(x, y)} \text { if } x \neq y
$$

for all $x, y \in G$, is a metric in the domain $G$.

Proof. Since $\eta_{G}$ is a metric and $c_{G}$ is both symmetric and non-negative, the function $\phi_{G}$ trivially fulfills the first two conditions of Definition 2.1. Note that, by the triangle inequality of the metric $\eta_{G}$ and the inequality (3.3), the inequalities

$$
\begin{aligned}
& \eta_{G}(x, y) \leq \eta_{G}(x, z)+\eta_{G}(z, y), \\
& c_{G}(x, z) \leq \eta_{G}(z, y)+c_{G}(x, y), \\
& c_{G}(z, y) \leq \eta_{G}(x, z)+c_{G}(x, y),
\end{aligned}
$$

hold for all $x, y, z \in G$. Now,

$$
\begin{aligned}
\phi_{G}(x, y) & =\frac{\eta_{G}(x, y)}{\eta_{G}(x, y)+c_{G}(x, y)} \leq \frac{\eta_{G}(x, z)+\eta_{G}(z, y)}{\eta_{G}(x, z)+\eta_{G}(z, y)+c_{G}(x, y)} \\
& \leq \frac{\eta_{G}(x, z)}{\eta_{G}(x, z)+c_{G}(x, z)}+\frac{\eta_{G}(z, y)}{\eta_{G}(z, y)+c_{G}(z, y)}=\phi_{G}(x, z)+\phi_{G}(z, y),
\end{aligned}
$$

so the function $\phi_{G}$ fulfills the triangle inequality and it must be a metric.

Remark 3.4. (1) If the function $c_{G}$ of Theorem 3.2 is strictly positive, the condition $\phi_{G}(x, x)=0$ does not need to be separately specified. Namely, this condition follows directly from the fact that $\eta_{G}(x, x)=0$ for a metric $\eta_{G}$. Note also that if $c_{G}$ is a null function, the function $\phi_{G}$ becomes the discrete metric. 
(2) If $\eta_{G}$ is a metric, then $\eta_{G}^{\alpha}$ is a metric, too, for $0<\alpha \leq 1$, but this is not true for $\alpha>1$ [7, Ex. 5.24, p. 80].

Corollary 3.5. The function $\psi: \mathbb{B}^{n} \times \mathbb{B}^{n} \rightarrow[0,1]$, defined as

$$
\psi(x, x)=0, \quad \psi(x, y)=\frac{|x-y|}{|x-y|+c|x||y|} \text { if } x \neq y,
$$

for all $x, y \in \mathbb{B}^{n}$ with a constant $0<c \leq 1$, is a metric on the unit ball.

Proof. Since now

$$
c|x|(|z|-|y|) \leq|| z|-| y|| \leq|z-y| \quad \Rightarrow \quad c|x||z| \leq|z-y|+c|x||y|,
$$

for all $x, y, z \in \mathbb{B}^{n}$, the result follows from Theorem 3.2.

Corollary 3.6. If $G$ is a proper subset of a metric space $X$ and $\eta_{G}$ is some metric defined in the closure of $G$ such that $\eta_{G}(x)=\inf \left\{\eta_{G}(x, u) \mid u \in \partial G\right\} \leq$ 1 for all $x \in G$, then a function $v_{G}: G \times G \rightarrow[0,1]$, defined as

$$
v_{G}(x, y)=\frac{\eta_{G}(x, y)}{\eta_{G}(x, y)+c \sqrt{\left(1+\eta_{G}(x)\right)\left(1+\eta_{G}(y)\right)}}
$$

with a constant $0<c \leq \sqrt{2}$ is a metric in the domain $G$.

Proof. Fix $c_{G}(x, y)=c \sqrt{\left(1+\eta_{G}(x)\right)\left(1+\eta_{G}(y)\right)}$. Now,

$$
\begin{aligned}
c_{G}(x, z)-c_{G}(x, y) & =c \sqrt{\left(1+\eta_{G}(x)\right)\left(1+\eta_{G}(z)\right)}-c \sqrt{\left(1+\eta_{G}(x)\right)\left(1+\eta_{G}(y)\right)} \\
& =c \cdot \frac{\left(1+\eta_{G}(x)\right)\left(1+\eta_{G}(z)\right)-\left(1+\eta_{G}(x)\right)\left(1+\eta_{G}(y)\right)}{\sqrt{\left(1+\eta_{G}(x)\right)\left(1+\eta_{G}(z)\right)}+\sqrt{\left(1+\eta_{G}(x)\right)\left(1+\eta_{G}(y)\right)}} \\
& =\frac{c \sqrt{1+\eta_{G}(x)}\left(\eta_{G}(z)-\eta_{G}(y)\right)}{\sqrt{1+\eta_{G}(z)}+\sqrt{1+\eta_{G}(y)}} \leq \frac{\sqrt{2} c\left(\eta_{G}(z)-\eta_{G}(y)\right)}{1+1} \\
& \leq \eta_{G}(z)-\eta_{G}(y) \leq \eta_{G}(z, y),
\end{aligned}
$$

so the inequality (3.3) holds for all $x, y, z \in G$ and the result follows from Theorem 3.2.

Corollary 3.7. The function $\chi: \mathbb{B}^{n} \times \mathbb{B}^{n} \rightarrow[0,1]$, defined as

$$
\chi(x, y)=\frac{|x-y|}{|x-y|+c \sqrt{(2-|x|)(2-|y|)}}
$$

for all $x, y \in \mathbb{B}^{n}$ with a constant $0<c \leq \sqrt{2}$, is a metric on the unit ball. Proof. Follows from Corollary 3.6.

Let us focus again on the $t$-metric. Since the result of Theorem 3.1 holds for any metric $\eta_{G}$, the $t$-metric is a metric when defined by using the Euclidean metric. Below, we will consider the $t$-metric in this special case only. Let us next prove the inequalities between the $t$-metric and the three hyperbolic type metrics defined earlier. 
Theorem 3.8. For all domains $G \subsetneq \mathbb{R}^{n}$ and all points $x, y \in G$, the following inequalities hold:

(1) $j_{G}^{*}(x, y) / 2 \leq t_{G}(x, y) \leq j_{G}^{*}(x, y)$,

(2) $p_{G}(x, y) / 2 \leq t_{G}(x, y) \leq p_{G}(x, y)$,

(3) $s_{G}(x, y) / 2 \leq t_{G}(x, y) \leq s_{G}(x, y)$.

Proof. (1) Trivially, $t_{G}(x, y) \leq j_{G}^{*}(x, y)$ and, by the triangle inequality,

$$
d_{G}(x)+d_{G}(y) \leq|x-y|+2 \min \left\{d_{G}(x), d_{G}(y)\right\},
$$

from which it follows that

$$
j_{G}^{*}(x, y) \leq \frac{2|x-y|}{|x-y|+2 \min \left\{d_{G}(x), d_{G}(y)\right\}+d_{G}(x)+d_{G}(y)} \leq 2 t_{G}(x, y) .
$$

(2) From Lemma 2.2 and Theorem 3.8(1), it follows that $t_{G}(x, y) \leq p_{G}(x, y)$. Let us now prove that $p_{G}(x, y) / 2 \leq t_{G}(x, y)$. This is clearly equivalent to

$$
|x-y|+d_{G}(x)+d_{G}(y) \leq 2 \sqrt{|x-y|^{2}+4 d_{G}(x) d_{G}(y)} .
$$

Fix $u=|x-y|, v=\min \left\{d_{G}(x), d_{G}(y)\right\}$ and $k=\left|d_{G}(x)-d_{G}(y)\right|$. The inequality (3.9) is now

$$
\begin{aligned}
& u+2 v+k \leq 2 \sqrt{u^{2}+4 v(v+k)} \\
& \quad \Leftrightarrow k^{2}+4 u v+2 u k-3 u^{2}-12 v^{2}-12 k v \leq 0 .
\end{aligned}
$$

Define a function $f(k)=k^{2}+4 u v+2 u k-3 u^{2}-12 v^{2}-12 k v$. Since the inequality above is equivalent to $f(k) \leq 0$, we need to find out the greatest value of this function. There is no upper limit for $u \geq 0$ or $v \geq 0$, but $0 \leq k \leq u$. We can solve that

$$
f^{\prime}(k)=2 k+2 u-12 v=0 \quad \Leftrightarrow \quad k=6 v-u .
$$

Since

$$
\begin{aligned}
f(0) & =-3 u^{2}+4 u v-12 v^{2} \leq-2 u^{2}-8 v^{2} \leq 0, \\
f(6 v-u) & =-4 u^{2}+16 u v-48 v^{2} \leq-32 v^{2} \leq 0, \\
f(u) & =-8 u v-12 v^{2} \leq 0,
\end{aligned}
$$

$f(k)$ is always non-positive on the closed interval $k \in[0, u]$ and, consequently, the inequality $p_{G}(x, y) / 2 \leq t_{G}(x, y)$ follows.

(3) By the triangle inequality and Lemma 2.3 and Theorem 3.8(1),

$$
\begin{aligned}
\frac{s_{G}(x, y)}{2} & \leq \frac{|x-y|}{\inf _{z \in \partial G}(|x-z|+|z-y|)+d_{G}(x)+d_{G}(y)} \\
& \leq t_{G}(x, y) \leq j_{G}^{*}(x, y) \leq s_{G}(x, y) .
\end{aligned}
$$


Proposition 3.10. For any fixed domain $G \subsetneq \mathbb{R}^{n}$, the inequalities of Theorem 3.8 are sharp.

Proof. For a proper subdomain $G$, there is some ball $B^{n}(x, r) \subset G$ with $S^{n-1}(x, r) \cap \partial G \neq \varnothing$ where $r>0$. Fix $z \in S^{n-1}(x, r) \cap \partial G$ and $y \in[x, z]$ so that $|y-z|=k r$ with $k \in(0,1)$. Clearly, $d_{G}(x)=r, d_{G}(y)=k r,|x-y|=(1-k) r$ and $\inf _{z \in \partial G}(|x-z|+|z-y|)=(1+k) r$. It follows that

$$
\begin{aligned}
& \lim _{k \rightarrow 0^{+}} \frac{t_{G}(x, y)}{j_{G}^{*}(x, y)}=\lim _{k \rightarrow 0^{+}} \frac{t_{G}(x, y)}{p_{G}(x, y)}=\lim _{k \rightarrow 0^{+}} \frac{t_{G}(x, y)}{s_{G}(x, y)}=\lim _{k \rightarrow 0^{+}}\left(\frac{1+k}{2}\right)=\frac{1}{2}, \\
& \lim _{k \rightarrow 1^{-}} \frac{t_{G}(x, y)}{j_{G}^{*}(x, y)}=\lim _{k \rightarrow 1^{-}} \frac{t_{G}(x, y)}{p_{G}(x, y)}=\lim _{k \rightarrow 1^{-}} \frac{t_{G}(x, y)}{s_{G}(x, y)}=\lim _{k \rightarrow 1^{-}}\left(\frac{1+k}{2}\right)=1 .
\end{aligned}
$$

Thus, regardless of how $G$ is chosen, the inequalities of Theorem 3.8 are sharp.

Next, we will study the connection between the $t$-metric and the hyperbolic metric.

Lemma 3.11. For all $x, y \in \mathbb{B}^{2}$, the inequality $|x-y|+2-|x|-|y| \leq 2 A[x, y]$ holds.

Proof. By the triangle inequality, $|x-y|^{2} \geq(|x|-|y|)^{2}$ holds and therefore

$$
A[x, y] \geq \sqrt{(|x|-|y|)^{2}+\left(1-|x|^{2}\right)\left(1-|y|^{2}\right)}=1-|x||y| .
$$

Thus, the inequality in the lemma holds if

$$
|x-y|+2-|x|-|y| \leq 2(1-|x||y|) \quad \Leftrightarrow \quad|x-y| \leq|x|+|y|-2|x||y| .
$$
$152]$,

Suppose then that $|x-y|>|x|+|y|-2|x||y|$. By [1, Lemma 7.57.(1),p.

$$
A[x, y] \geq|x-y|+(1-|x|)(1-|y|)
$$

for all $x, y \in \mathbb{B}^{2}$. Because

$$
\begin{aligned}
& |x-y|+2-|x|-|y| \leq 2|x-y|+2(1-|x|)(1-|y|) \\
& \quad \Leftrightarrow|x-y| \geq|x|+|y|-2|x||y|,
\end{aligned}
$$

the inequality of the lemma holds in this case, too.

Theorem 3.12. For all $x, y \in G \in\left\{\mathbb{H}^{n}, \mathbb{B}^{n}\right\}$, the inequality

$$
\frac{1}{2} \operatorname{th} \frac{\rho_{G}(x, y)}{2} \leq t_{G}(x, y) \leq \operatorname{th} \frac{\rho_{G}(x, y)}{2}
$$

holds and the constants here are sharp.

Proof. If $G=\mathbb{H}^{n}$, the inequality follows directly from Lemma 2.4(1) and Theorem 3.8(3), and its sharpness from Proposition 3.10. By Lemma 2.4(2) and Theorem 3.8(1),

$$
t_{\mathbb{B}^{n}}(x, y) \leq j_{\mathbb{B}^{n}}^{*}(x, y) \leq \operatorname{th} \frac{\rho_{\mathbb{B}^{n}}(x, y)}{2}
$$


for all $x, y \in \mathbb{B}^{n}$ and this is sharp because $x=k e_{1}$ and $y=-k e_{1}$ fulfill $\lim _{k \rightarrow 1^{-}}\left(t_{\mathbb{B}^{n}}(x, y) / \operatorname{th}\left(\rho_{\mathbb{B}^{n}}(x, y) / 2\right)\right)=1$. By Lemma 3.11 ,

$$
\frac{t_{\mathbb{B}^{2}}(x, y)}{\operatorname{th}\left(\rho_{\mathbb{B}^{2}}(x, y) / 2\right)}=\frac{A[x, y]}{|x-y|+2-|x|-|y|} \geq \frac{1}{2} .
$$

Since the values of the $t$-metric and the hyperbolic metric in the domain $\mathbb{B}^{n}$ only depend on how the points $x, y$ are located on the two-dimensional plane containing these two points and the origin, the inequality above holds also in the general case where $n \geq 2$. This inequality is sharp: For $x=k e_{1}$ and $y=-k e_{1}, \lim _{k \rightarrow 0^{+}}\left(t_{\mathbb{B}^{n}}(x, y) / \operatorname{th}\left(\rho_{\mathbb{B}^{n}}(x, y) / 2\right)\right)=1 / 2$.

Theorem 3.13. For a fixed angle $\theta \in(0,2 \pi)$ and for all $x, y \in S_{\theta}$, the following inequalities hold:

(1) $t_{S_{\theta}}(x, y) \leq \operatorname{th}\left(\rho_{S_{\theta}}(x, y) / 2\right) \leq 2(\pi / \theta) \sin (\theta / 2) t_{S_{\theta}}(x, y)$ if $\theta \in(0, \pi)$,

(2) $t_{S_{\theta}}(x, y) \leq \operatorname{th}\left(\rho_{S_{\theta}}(x, y) / 2\right) \leq 2 t_{S_{\theta}}(x, y)$ if $\theta=\pi$,

(3) $(\pi / \theta) t_{S_{\theta}}(x, y) \leq \operatorname{th}\left(\rho_{S_{\theta}}(x, y) / 2\right) \leq 2 t_{S_{\theta}}(x, y)$ if $\theta \in(\pi, 2 \pi)$.

Proof. Follows from Theorems 3.8(3) and 3.12, and [14, Cor. 4.9, p. 9].

\section{Quasiconformal Mappings and Lipschitz Constants}

In this section, we will study the behaviour of the $t$-metric under different conformal and quasiconformal mappings in order to demonstrate how this metric works.

Remark 4.1. The $t$-metric is invariant under all similarity maps. In particular, the $t$-metric defined in a sector $S_{\theta}$ is invariant under a reflection over the bisector of the sector and a stretching $x \mapsto r \cdot x$ with any $r>0$. Consequently, this allows us to make certain assumptions when choosing the points $x, y \in S_{\theta}$.

First, let us study how the $t$-metric behaves under a $K$-quasiconformal homeomorphism between two sectors, see [15, Ch. 2] for the definition of this type of mappings. Let $c(K)$ be as in $[7$, Thm 16.39 , p. 313]

$$
c(K) \leq v(K-1)+K, \quad v=\log \left(2\left(1+\sqrt{1-1 / e^{2}}\right)\right)<1.3507 .
$$

Now, $c(K) \geq K$ and $c(K) \rightarrow 1$ whenever $K \rightarrow 1$. See also the book [5] by F.W. Gehring and K. Hag.

Theorem 4.2. If $\alpha, \beta \in(0,2 \pi)$ and $f: S_{\alpha} \rightarrow S_{\beta}=f\left(S_{\alpha}\right)$ is a $K$ quasiconformal homeomorphism, the following inequalities hold for all $x, y \in$ $S_{\alpha}$. 
(1)

$$
\begin{aligned}
& \frac{\beta}{2 c(K)^{K} \pi \sin (\beta / 2)} t_{S_{\alpha}}(x, y)^{K} \leq t_{S_{\beta}}(f(x), f(y)) \\
& \leq c(K)\left(\frac{\pi}{\alpha} \sin \left(\frac{\alpha}{2}\right)\right)^{1 / K} t_{S_{\alpha}}(x, y)^{1 / K} \\
& \text { if } \alpha, \beta \in(0, \pi]
\end{aligned}
$$

$$
\begin{aligned}
& \frac{1}{2 c(K)^{K}} t_{S_{\alpha}}(x, y)^{K} \leq t_{S_{\beta}}(f(x), f(y)) \\
& \leq \frac{c(K) \beta}{\pi}\left(\frac{\pi}{\alpha} \sin \left(\frac{\alpha}{2}\right)\right)^{1 / K} t_{S_{\alpha}}(x, y)^{1 / K}
\end{aligned}
$$

if $\alpha \in(0, \pi)$ and $\beta \in(\pi, 2 \pi)$,

(3) $\frac{1}{2}\left(\frac{\pi}{c(K) \alpha}\right)^{K} t_{S_{\alpha}}(x, y)^{K} \leq t_{S_{\beta}}(f(x), f(y)) \leq \frac{c(K) \beta}{\pi} t_{S_{\alpha}}(x, y)^{1 / K}$ if $\alpha, \beta \in[\pi, 2 \pi)$.

Proof. Follows from Theorem 3.8(3) and [14, Cor. 5.7, p. 12].

Let us now find Lipschitz constants of a few different mappings for the $t$-metric.

Theorem 4.3. For all conformal mappings $f: G_{1} \rightarrow G_{2}=f\left(G_{1}\right)$ with $G_{1}, G_{2} \in\left\{\mathbb{H}^{n}, \mathbb{B}^{n}\right\}$, the inequality

$$
\frac{1}{2} t_{G_{1}}(x, y) \leq t_{G_{2}}(f(x), f(y)) \leq 2 t_{G_{1}}(x, y)
$$

holds for all $x, y \in G_{1}$.

Proof. Follows from Theorem 3.12 and the conformal invariance of the hyperbolic metric.

It follows from Theorem 4.3 that the Lipschitz constant $\operatorname{Lip}\left(f \mid G_{1}\right)$ for the $t$-metric in any conformal mapping $f: G_{1} \rightarrow G_{2}=f\left(G_{1}\right), G_{1}, G_{2} \in\left\{\mathbb{H}^{n}, \mathbb{B}^{n}\right\}$, is at most 2. Suppose now that $h$ is the Möbius transformation $h: \mathbb{B}^{2} \rightarrow \mathbb{H}^{2}$, $h(z)=(1-z) i /(1+z)$. Since, for $x=0$ and $y=\frac{1-k}{k+1}$ with $0<k<1$,

$$
\lim _{k \rightarrow 1^{-}}\left(\frac{t_{\mathbb{H}^{n}}(h(x), h(y))}{t_{\mathbb{B}^{n}}(x, y)}\right)=\lim _{k \rightarrow 1^{-}}(k+1)=2,
$$

the Lipschitz constant $\operatorname{Lip}\left(h \mid \mathbb{B}^{2}\right)$ is equal to 2 . However, for certain fixed choices of the Möbius transformation $h$, there is an even better Lipschitz constant $\operatorname{Lip}\left(h \mid \mathbb{B}^{2}\right)$ than 2 . For instance, the following conjecture is supported by several numerical tests.

Conjecture 4.4. For all $a, x, y \in \mathbb{B}^{2}$, the Möbius transformation $T_{a}: \mathbb{B}^{2} \rightarrow \mathbb{B}^{2}$, $T_{a}(z)=(z-a) /(1-\bar{a} z)$ fulfills the inequality

$$
t_{\mathbb{B}^{2}}\left(T_{a}(x), T_{a}(y)\right) \leq(1+|a|) t_{\mathbb{B}^{2}}(x, y) .
$$


Remark 4.5. It is also an open question whether the inequality of Conjecture 4.4 holds for the triangular ratio metric or so called Barrlund metric, but numerical tests suggest so, see [3, Conj. 1.6, p. 684] and [4, Conj. 4.3, p. 25].

In the next few results, we will study a mapping $f^{*}: S_{\theta} \rightarrow S_{\theta}, f^{*}(x)=x /$ $|x|^{2}$ defined in some open sector $S_{\theta}$, and find its Lipschitz constants for the $t$-metric.

Theorem 4.6. If $\theta \in(0, \pi]$ and $f^{*}$ is the mapping $f^{*}: S_{\theta} \rightarrow S_{\theta}, f^{*}(x)=x /$ $|x|^{2}$, the Lipschitz constant $\operatorname{Lip}\left(f^{*} \mid S_{\theta}\right)$ for the $t$-metric is $1+\sin (\theta / 2)$.

Proof. Without loss of generality, we can fix $x=e^{h i}$ and $y=r e^{k i}$ with $0<$ $h \leq \theta / 2, h \leq k<\theta$ and $r>0$. Since $x^{*}=e^{h i}$ and $y^{*}=(1 / r) e^{k i}$, it follows that

$$
\frac{t_{S_{\theta}}\left(x^{*}, y^{*}\right)}{t_{S_{\theta}}(x, y)}=\frac{\sqrt{1+r^{2}-2 r \cos (k-h)}+\sin (h)+r \sin (\min \{k, \theta-k\})}{\sqrt{1+r^{2}-2 r \cos (k-h)}+r \sin (h)+\sin (\min \{k, \theta-k\})} .
$$

To maximize this, we clearly need to choose $k=\theta / 2$ and make $r$ and $h$ as small as possible. If $k=\theta / 2$,

$$
\lim _{h \rightarrow 0^{+}, r \rightarrow 0^{+}} \frac{t_{S_{\theta}}\left(x^{*}, y^{*}\right)}{t_{S_{\theta}}(x, y)}=1+\sin (\theta / 2),
$$

so the theorem follows.

Theorem 4.7. If $x^{*}=x /|x|^{2}$ and $y^{*}=y /|y|^{2}$, the equality $s_{S_{\theta}}(x, y)=$ $s_{S_{\theta}}\left(x^{*}, y^{*}\right)$ holds in an open sector $S_{\theta}$ with $\theta \in(0,2 \pi)$.

Proof. Fix $x=e^{h i}$ and $y=r e^{k i}$ where $r>0$ and $0<h \leq k<\theta$. Clearly, $x^{*}=$ $x=e^{h i}$ and $y^{*}=(1 / r) e^{k i}$. Suppose first that $\theta \leq \pi$. By the known solution to Heron's problem, the infimum $\inf _{z \in \partial S_{\theta}}(|x-z|+|z-y|)$ is $\min \left\{|\bar{x}-y|,\left|x-y^{\prime}\right|\right\}$, where $y^{\prime}$ is the point $y$ reflected over the left side of the sector $\theta$. Clearly,

$$
\begin{aligned}
& \quad|\bar{x}-y| \leq\left|x-y^{\prime}\right| \quad \Leftrightarrow \quad\left|e^{-h i}-r e^{k i}\right| \leq\left|e^{h i}-r e^{(2 \theta-k) i}\right| \\
& \Leftrightarrow \quad\left|1-r e^{(h+k) i}\right| \leq\left|1-r e^{(2 \theta-h-k) i}\right| \quad \Leftrightarrow \quad h+k \leq 2 \theta-h-k \\
& \Leftrightarrow \quad(h+k) / 2 \leq \theta / 2 .
\end{aligned}
$$

By symmetry, we may assume that $(h+k) / 2 \leq \theta / 2$ without loss of generality. Note that it follows from above that not only $\inf _{z \in \partial S_{\theta}}(|x-z|+\mid z-$

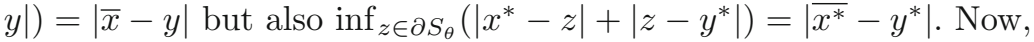

$$
\begin{aligned}
s_{S_{\theta}}(x, y) & =\frac{|x-y|}{|\bar{x}-y|}=\frac{\left|1-r e^{(k-h) i}\right|}{\left|1-r e^{(k+h) i}\right|}=\frac{\left|1-r e^{(h-k) i}\right|}{\left|1-r e^{-(k+h) i}\right|}=\frac{\left|r-e^{(k-h) i}\right|}{\left|r-e^{(k+h) i}\right|} \\
& =\frac{\left|1-(1 / r) e^{(k-h) i}\right|}{\left|1-(1 / r) e^{(k+h) i}\right|}=\frac{\left|x^{*}-y^{*}\right|}{\left|\overline{x^{*}}-y^{*}\right|}=s_{S_{\theta}}\left(x^{*}, y^{*}\right) .
\end{aligned}
$$


Consider now the case where $\theta>\pi$. If $k-h \geq \pi$, then $s_{S_{\theta}}(x, y)=1=$ $s_{S_{\theta}}\left(x^{*}, y^{*}\right)$ always so suppose that $k-h<\pi$ instead. This leaves us three possibilities. If $(h+k) / 2 \leq \pi / 2$, then $(h+k) / 2<\theta / 2$ and

$$
s_{S_{\theta}}(x, y)=\frac{|x-y|}{|\bar{x}-y|}=\frac{\left|x^{*}-y^{*}\right|}{\left|\overline{x^{*}}-y^{*}\right|}=s_{S_{\theta}}\left(x^{*}, y^{*}\right),
$$

just like above. By symmetry, $s_{S_{\theta}}(x, y)=s_{S_{\theta}}\left(x^{*}, y^{*}\right)$ also if $(k+h) /$ $2 \geq \theta-\pi / 2$. If $\pi / 2<(k+h) / 2<\theta-\pi / 2$ instead, then

$$
\begin{aligned}
s_{S_{\theta}}(x, y) & =\frac{|x-y|}{|x|+|y|}=\frac{\left|1-r e^{(k-h) i}\right|}{1+r}=\frac{\left|1-(1 / r) e^{(k-h) i}\right|}{1+1 / r} \\
& =\frac{\left|x^{*}-y^{*}\right|}{\left|x^{*}\right|+\left|y^{*}\right|}=s_{S_{\theta}}\left(x^{*}, y^{*}\right) .
\end{aligned}
$$

Theorem 4.8. If $\theta \in[\pi, 2 \pi)$ and $f^{*}: S_{\theta} \rightarrow S_{\theta}$ is the mapping $f^{*}(x)=x /|x|^{2}$, the Lipschitz constant Lip $\left(f^{*} \mid S_{\theta}\right)$ for the t-metric is 2.

Proof. It follows from Theorems 4.7 and 3.8(3) that

$$
\frac{t_{S_{\theta}}(x, y)}{2} \leq t_{S_{\theta}}\left(x^{*}, y^{*}\right) \leq 2 t_{S_{\theta}}(x, y)
$$

for all $x, y \in S_{\theta}$. Since for $x=e^{h i}$ and $y=r e^{\pi i / 2}$ with $h<\pi / 2$ and $r>0$,

$\lim _{h, r \rightarrow 0^{+}} \frac{t_{S_{\theta}}\left(x^{*}, y^{*}\right)}{t_{S_{\theta}}(x, y)}=\lim _{h, r \rightarrow 0^{+}}\left(\frac{\sqrt{1+r^{2}-2 r \cos (\pi / 2-h)}+r \sin (h)+1}{\sqrt{1+r^{2}-2 r \cos (\pi / 2-h)}+\sin (h)+r}\right)=2$, and it follows that

$$
\sup \left\{\frac{t_{S_{\theta}}\left(x^{*}, y^{*}\right)}{t_{S_{\theta}}(x, y)} \mid x, y \in S_{\theta}, x \neq y, \theta \in[\pi, 2 \pi)\right\}=2 \text {. }
$$

\section{Comparison of Metric Balls}

Next, we will graphically demonstrate the differences and similarities between the various metrics considered in this paper by drawing boundaries of balls centered at the same point but with different radii for each metric. In all of the figures of this section, the domain $G \subset \mathbb{R}^{2}$ is a regular five-pointed star and the circles have the radii $r=1 / 10, \ldots, 9 / 10$. The center of these circles is in the center of $G$ in the first figures, and then off the center in the rest of the figures. All the figures in this section were drawn by using the contour plot function contour in R-Studio and choosing a grid of the size 1,000×1,000 test points. While we graphically only inspect circles and disks, we will also prove some properties for the $n$-dimensional metric balls. 


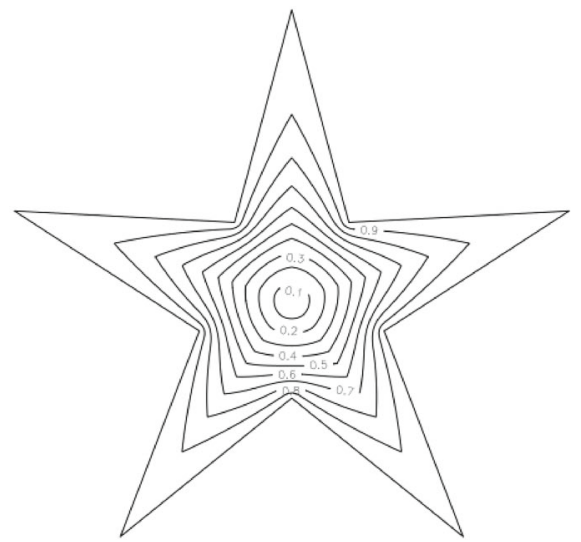

(A) $s_{G}$-metric circles.

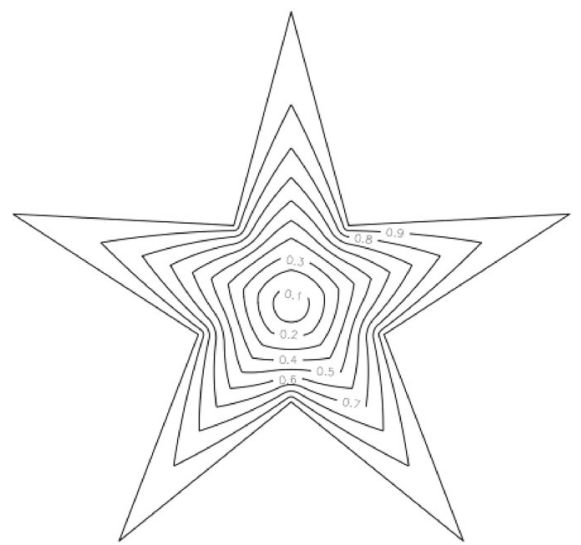

(c) $p_{G}$-metric circles.

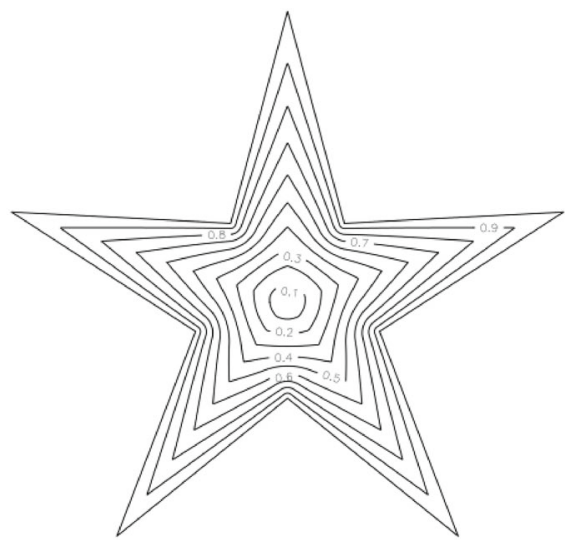

(B) $j_{G}^{*}$-metric circles.

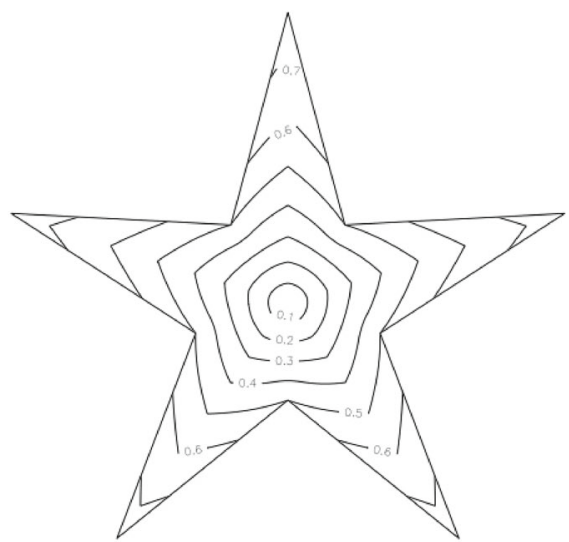

(D) $t_{G}$-metric circles.

Figure 1. Circles in a five-pointed star domain with different metrics

For several hyperbolic type metrics, the metric balls of small radii resemble Euclidean balls, but the geometric structure of the boundary of the domain begins to affect the shape of these balls when their radii grow large enough, see [7, Ch. 13, pp. 239-259]. By analysing this phenomenon more carefully, we can observe, for instance, that the balls are convex with radii less than some fixed $r_{0}>0$ in the case of some other metrics, see [7, Thm 13.6, p. 241; Thm 13.41 p. 256; Thm 13.44, p. 258]. From the figures of this section, we see that the four metrics studied here share this same property. In particular, we notice that, while the metric disks with small radii are convex and round like Euclidean disks, the metric circles with larger radii are non-convex and have 
corner points. By a corner point, we mean here such a point on the circle arc where no tangent exists.

In the following theorem, we will prove a property that can be seen from Figs. 1c, d and 2c, d.

Theorem 5.1. If the domain $G$ is a polygon, then the corner points of the circles $S_{p}(x, r)$ and $S_{t}(x, r)$ are located on the angle bisectors of $G$.

Proof. Suppose $G$ has sides $l_{0}$ and $l_{1}$ that have a common endpoint $k$. Fix $x \in G$ and choose some point $y \in G$ so that $k$ is the vertex of $G$ that is closest to $y$ and there is no other side closer to $y$ than $l_{0}$ and $l_{1}$. Thus, $d_{G}(y)=$ $\min \left\{d\left(y, l_{0}\right), d\left(y, l_{1}\right)\right\}$ and, for a fixed distance $|x-y|, d_{G}(y)$ is at maximum when $d\left(y, l_{0}\right)=d\left(y, l_{1}\right)$. The condition $d\left(y, l_{0}\right)=d\left(y, l_{1}\right)$ is clearly fulfilled when $y$ is on the bisector of $\angle\left(l_{0}, l_{1}\right)$ and, the greater the $d_{G}(y)$, the smaller the distances $p_{G}(x, y)$ and $t_{G}(x, y)$ are now. Consequently, if the circle $S_{p}(x, r)$ or $S_{t}(x, r)$ has a corner point, it must be located on an angle bisector of $G$.

However, it can been seen from Fig. 2a, b that the circles with the metrics $s_{G}$ and $j_{G}^{*}$ can have corner points also elsewhere than on the angle bisectors of the domain $G$. We also notice that the circles in Fig. 2b clearly differ from those in Fig. 2a. This can be described with the concept of starlikeness, which is a looser form of convexity. Namely, a set $K$ is starlike with respect to a point $x \in K$ if and only if the segment $[x, y]$ belongs to $K$ fully for every $y \in K$. In particular, the five-pointed star domain is starlike with respect to its center. The disks by the metrics $j_{G}^{*}$ and $t_{G}$ (Fig. 2b, d) are clearly not starlike and, even if it cannot be clearly seen from Fig. 2c, there are disks drawn with the point pair function $p_{G}$ that are not starlike.

Lemma 5.2. There exist disks $B_{j^{*}}(x, r), B_{p}(x, r)$, and $B_{t}(x, r)$ that are not starlike with respect to their center.

Proof. Consider the domain $G=\mathbb{H}^{2} \cup\{z \in \mathbb{C} \mid-1<\operatorname{Re}(z)<1,-3<$ $\operatorname{Im}(z) \leq 0\}$. Fix $x=-2 i$ and $y=3+i$. Clearly, $d_{G}(x)=d_{G}(y)=1$ and $|x-y|=3 \sqrt{2}$. Consequently,

$$
j_{G}^{*}(x, y)=t_{G}(x, y)=\frac{3}{3+\sqrt{2}}<0.7, \quad p_{G}(x, y)=\frac{3}{\sqrt{11}}<0.91 .
$$

The segment $[x, y]$ does not clearly belong to $G$ fully and no disk in $G$ can contain this segment. However, its end point $y$ is clearly included in the disks $B_{j^{*}}(x, 0.7), B_{t}(x, 0.7)$ and $B_{p}(x, 0.91)$. Thus, we have found examples of non-starlike disks.

There are no disks or balls like this for the triangular ratio metric.

Lemma 5.3. [\%, p. 206] The balls $B_{s}(x, r)$ in any domain $G \subsetneq \mathbb{R}^{n}$ are always starlike with respect to their center $x$. 


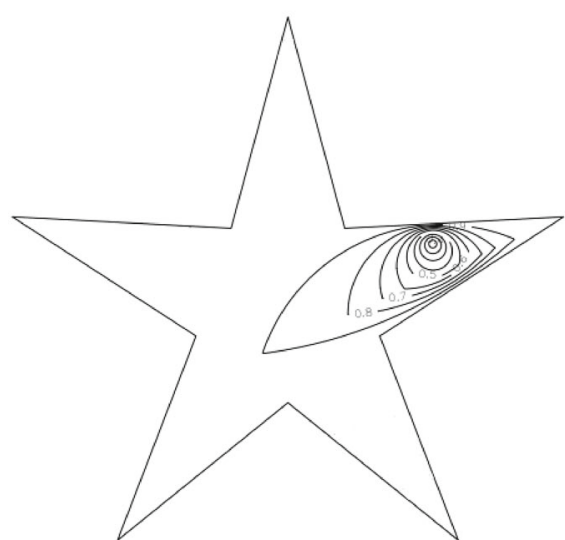

(A) $s_{G}$-metric circles.

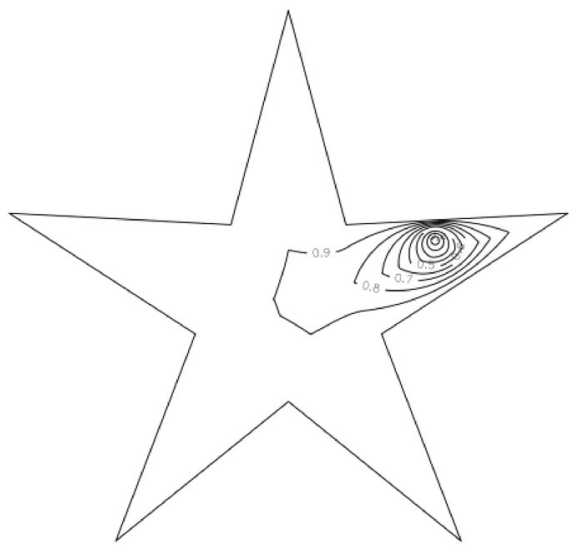

(C) $p_{G^{-}}$-metric circles.

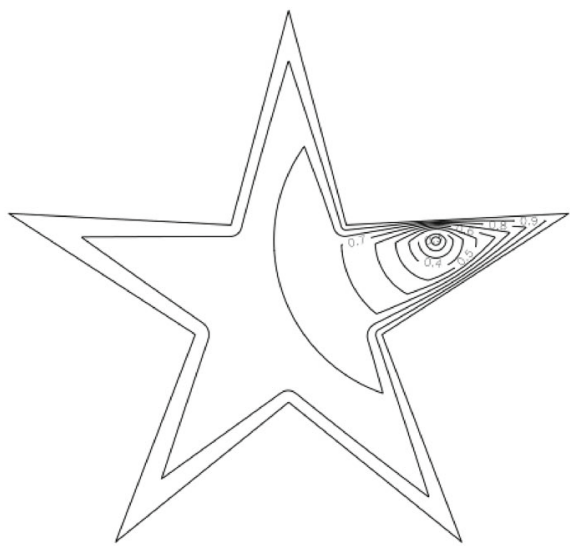

(в) $j_{G}^{*}$-metric circles.

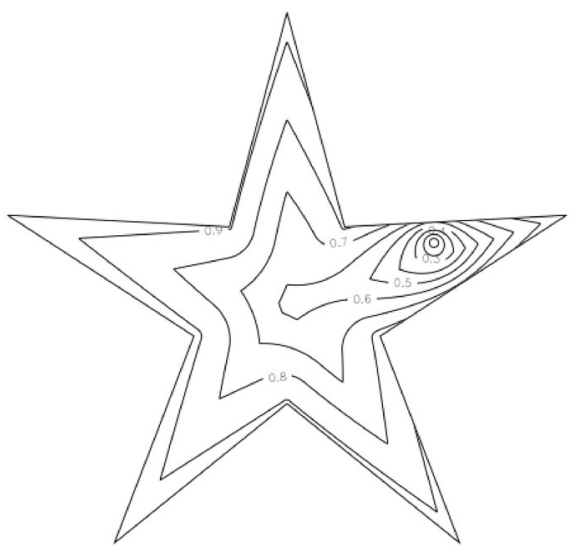

(D) $t_{G}$-metric circles.

Figure 2. Circles in a five-pointed star domain with different metrics

For several common hyperbolic type metrics $\eta_{G}$, the closed ball $\overline{B_{\eta}(x, M)}$ with $M=\eta_{G}(x, y)$ and $x, y \in G$ is always a compact subset of the domain $G$, see [7, p. 79]. For instance, the hyperbolic metric $\rho_{G}$ has this property $[7$, p. 192]. As can be seen from the figures, the $j^{*}$-metric, the triangular ratio metric and the point pair function share this property, too.

Lemma 5.4. The balls $B_{j^{*}}(x, r), B_{p}(x, r)$ and $B_{s}(x, r)$ touch the boundary of the domain $G \subsetneq \mathbb{R}^{n}$ if and only if $r=1$.

Proof. If the ball $B_{\eta}(x, r), \eta_{G} \in\left\{j_{G}^{*}, p_{G}, s_{G}\right\}$, touches the boundary of $G$, then there is some point $y \in B_{\eta}(x, r)$ with $d_{G}(y) \rightarrow 0^{+}$and $\eta_{G}(x, y) \rightarrow 1^{-}$. Thus, we need to just prove that the balls with radius 1 always touch the 
boundary. Consider then the line segment $[x, z]$ between a point $x \in G$ and a point $z \in \partial G$ such that $|x-z|=d_{G}(x)$. For $\eta_{G} \in\left\{j_{G}^{*}, p_{G}\right\}$, the ball $B_{\eta}(x, 1)$ must include all the points $[x, z] \cap G$ because $\eta_{G}(x, y)<1$ by definition for all $y \in G$, and thus there is some point $y \in[x, z]$ with $d_{G}(y) \rightarrow 0^{+}$. The triangular ratio metric $s_{G}(x, y)$ is 1 if and only if $|x-y|=|x-u|+|u-y|$ for some $u \in \partial G$ or, equivalently, $[x, y] \cap \partial G \neq \varnothing$. However, if $|x-z|=d_{G}(x)$, then $[x, z] \cap \partial G=\{z\}$ and, for all points $y \in[x, z] \cap G, s_{G}(x, y)<1$. Thus, there is some point $y \in[x, z]$ with $d_{G}(y) \rightarrow 0^{+}$and this point $y$ is included in the ball $B_{s}(x, 1)$.

However, the $t$-metric differs from the hyperbolic type metrics in this aspect: the closure of a $t$-metric ball is a compact set, if and only if the radius of the ball is less than $1 / 2$.

Theorem 5.5. The balls $B_{t}(x, r)$ touch the boundary of the domain $G \subsetneq \mathbb{R}^{n}$ if and only if $r \geq \frac{1}{2}$.

Proof. If $B_{t}(x, r)$ touches the boundary, there must be some $y \in B_{t}(x, r)$ such that $d_{G}(y) \rightarrow 0^{+}$. Since $d_{G}(x) \leq|x-y|+d_{G}(y)$, it follows that $t_{G}(x, y)$ is equal to or greater than the limit value

$$
\frac{|x-y|}{|x-y|+|x-y|+0+0}=\frac{1}{2} \text {. }
$$

Thus, only balls $B_{t}(x, r)$ with a radius $r \geq \frac{1}{2}$ can touch the boundary of $G$.

Let us yet prove that the balls $B_{t}\left(x, \frac{1}{2}\right)$ always touch the boundary of $G$. Fix $x \in G$ and $z \in \partial G$ so that $|x-z|=d_{G}(x)$. For all points $y \in[x, z] \cap G$,

$$
t_{G}(x, y)=\frac{|x-y|}{|x-y|+d_{G}(x)+d_{G}(x)-|x-y|}=\frac{|x-y|}{2 d_{G}(x)}<\frac{1}{2} .
$$

Thus, there is some point $y \in[x, z]$ with $d_{G}(y) \rightarrow 0^{+}$and it is included in the ball $B_{t}\left(x, \frac{1}{2}\right)$.

The result above is visualized in Figs. $1 \mathrm{~d}$ and $2 \mathrm{~d}$.

\section{Acknowledgements}

The authors are thankful to the referee for their detailed corrections

Funding Open Access funding provided by University of Turku (UTU) including Turku University Central Hospital. The research of the first author was supported by Finnish Concordia Fund 
Availability of data and code not applicable

\section{Declarations}

conflict of interest On behalf of all authors, the corresponding author states that there is no conflict of interest

Open Access. This article is licensed under a Creative Commons Attribution 4.0 International License, which permits use, sharing, adaptation, distribution and reproduction in any medium or format, as long as you give appropriate credit to the original author(s) and the source, provide a link to the Creative Commons licence, and indicate if changes were made. The images or other third party material in this article are included in the article's Creative Commons licence, unless indicated otherwise in a credit line to the material. If material is not included in the article's Creative Commons licence and your intended use is not permitted by statutory regulation or exceeds the permitted use, you will need to obtain permission directly from the copyright holder. To view a copy of this licence, visit http://creativecommons.org/ licenses/by/4.0/.

\section{References}

[1] Anderson,G., Vamanamurthy,M., Vuorinen,M.: Conformal Invariants, Inequalities, and Quasiconformal Maps. Wiley, 1997

[2] Beardon,A.F., Minda,D.: The hyperbolic metric and geometric function theory. In: Ponnusamy, S., Sugawa, T., Vuorinen, M. (eds.) Proceedings of International Workshop on Quasiconformal Mappings and their Applications (IWQCMA05), pp. 9-56 (2006)

[3] Chen, J., Hariri, P., Klén, R., Vuorinen, M.: Lipschitz conditions, triangular ratio metric, and quasiconformal maps. Ann. Acad. Sci. Fenn. Math. 40, 683709 (2015)

[4] Fujimura, M., Mocanu, M., Vuorinen, M.: Barrlund's distance function and quasiconformal maps. Complex Var. Elliptic Equ. 66, 1225-1255 (2021)

[5] Gehring,F.W., Hag,K.: The ubiquitous quasidisk. With contributions by Ole Jacob Broch. Mathematical Surveys and Monographs, 184. American Mathematical Society, Providence, RI, 2012

[6] Gehring, F.W., Osgood, B.G.: Uniform domains and the quasi-hyperbolic metric. J. Anal. Math. 36, 50-74 (1979)

[7] Hariri,P., Klén, R., Vuorinen, M.: Conformally Invariant Metrics and Quasiconformal Mappings. Springer (2020)

[8] Hariri, P., Vuorinen, M., Zhang, X.: Inequalities and bilipschitz conditions for triangular ratio metric. Rocky Mount. J. Math. 47(4), 1121-1148 (2017)

[9] Hästö, P.: A new weighted metric, the relative metric I. J. Math. Anal. Appl. 274, 38-58 (2002)

[10] Ibragimov, Z., Mohapatra, M., Sahoo, S., Zhang, X.: Geometry of the Cassinian metric and its inner metric. Bull. Malays. Math. Sci. Soc. 40(1), 361-372 (2017) 
[11] Mohapatra, M., Sahoo, S.: A Gromov hyperbolic metric vs the hyperbolic and other related metrics. Comput. Methods Funct. Theory 18(3), 473-493 (2018)

[12] Rainio, O.: Intrinsic quasi-metrics. Bull. Malays. Math. Sci. Soc. 44, 2873-2891 (2021)

[13] Rainio,O., Vuorinen,M.: Triangular ratio metric in the unit disk. Complex Var. Elliptic Equ. (to appear). https://doi.org/10.1080/17476933.2020.1870452

[14] Rainio, O., Vuorinen, M.: Triangular Ratio Metric Under Quasiconformal Mappings In Sector Domains. Comput. Methods Func. Theory (to appear) Arxiv, 2005.11990

[15] Väisälä,J.: Lectures on $n$-dimensional quasiconformal mappings. Lecture Notes in Math, Vol. 229. Springer (1971)

Oona Rainio and Matti Vuorinen

Department of Mathematics and Statistics

University of Turku

20014 Turku

Finland

e-mail: ormrai@utu.fi;

vuorinen@utu.fi

Received: June 4, 2021.

Accepted: December 22, 2021.

Publisher's Note Springer Nature remains neutral with regard to jurisdictional claims in published maps and institutional affiliations. 